\title{
Circadian Expression of the dnaK Gene in the Cyanobacterium Synechocystis sp. Strain PCC 6803
}

\author{
SETSUYUKI AOKI, TAKAO KONDO, AND MASAHIRO ISHIURA* \\ National Institute for Basic Biology, Okazaki, Aichi 444, Japan
}

Received 6 February 1995/Accepted 24 July 1995

\begin{abstract}
The expression of the dnaK gene in the cyanobacterium Synechocystis sp. strain PCC 6803 was continuously monitored as bioluminescence by an automated monitoring system, using the bacterial luciferase genes (luxAB) of Vibrio harveyi as a reporter of promoter activity. A dnaK-reporting bioluminescent Synechocystis strain was constructed by fusing a promoterless segment of the lux $A B$ gene set downstream of the promoter region of the Synechocystis dnaK gene and introduction of this gene fusion into a BglII site downstream of the $n d h B$ gene in the Synechocystis chromosome. Bioluminescence from this strain was continuously monitored and oscillated with a period of about $22 \mathrm{~h}$ for at least 5 days in continuous light. The phase of the rhythm was reset by the timing of the 12-h dark period administered prior to the continuous light. The period of the rhythm was temperature compensated between 25 and $35^{\circ} \mathrm{C}$. Thus, the bioluminescence rhythm satisfied the three criteria of circadian rhythms. Furthermore, the abundance of dnaK mRNA also oscillated with a period of about 1 day for at least 2 days in continuous light conditions, indicating circadian control of dnaK gene expression in Synechocystis sp. strain PCC 6803.
\end{abstract}

Circadian rhythms are found ubiquitously in organisms from cyanobacteria to humans and are thought to be an adaptation to daily changes in environmental conditions on the earth, such as light and temperature (4), although the molecular mechanism that generates the rhythms (circadian clock) remains to be solved. Temperature is lowest just before dawn and highest about noon. Circadian rhythms of heat resistance have been observed in many plants $(12,18,26,32)$. Consistent with temperature change, heat resistance is maximal about noon and minimal during the night $(12,18)$. Heat stress transiently increases the rate of synthesis of heat shock proteins in various organisms from Escherichia coli to humans (8), and the proteins may be directly involved in acquisition of heat resistance $(11,17,30,39)$. The heat inducibility of heat shock protein synthesis is controlled by a circadian clock in Neurospora crassa (7), pea (Pisum sativum L. [24]), and cotton (Gossypium hirsutum L. [26]). Accumulation of mRNAs for several heat shock proteins in P. sativum L. is also under the control of the clock (24).

DnaK is one member of a well-conserved group of heat shock proteins and plays a protective role in supporting growth at high temperatures beyond the normal physiological range of E. coli (16). DnaK, DnaJ, and GrpE are known to be key proteins involved in regulation of heat shock gene expression in $E$. coli $(8,9,34,36)$. Thus, whether the expression of the dnaK gene is under the control of a circadian clock is an interesting question.

In previous studies, we monitored the expression of the $p s b I$ gene, which encodes D1, a major protein component of the photosystem II reaction center, in the unicellular cyanobacterium Synechococcus sp. strain PCC 7942 by using the bacterial luciferase $\operatorname{lu} x A B$ gene set from Vibrio harveyi as a reporter. We found that the expression of the $p s b A I$ gene, monitored as bioluminescence, is controlled by a circadian clock in this Synechococcus strain (13-15).

\footnotetext{
* Corresponding author. Present address: Department of Biology, Faculty of Science, Nagoya University, Furo-cho, Chikusa-ku, Nagoya 464-01, Japan. Phone: 81-52-789-2495. Fax: 81-52-789-2963. Electronic mail address: ishiura@bio.nagoya-u.ac.jp.
}

We took advantage of the fact that the dnaK gene has been isolated from the unicellular cyanobacterium Synechocystis sp. strain PCC 6803 and characterized (6) to monitor the expression of the $d n a K$ gene in this study. Another advantage of using this Synechocystis strain for circadian studies is that there is a glucose-tolerant derivative of the Synechocystis strain which is able to carry out light-activated heterotrophic growth in glucose-supplemented medium in near darkness (1). As in the case of the Synechococcus strain used previously (14), the Synechocystis strain also has a naturally occurring gene transfer system and is amenable to molecular analyses (38). We fused a promoterless segment of the $\operatorname{lu} x A B$ gene set downstream of the promoter segment of the Synechocystis dnaK gene and introduced the gene fusion into a specific site of the Synechocystis chromosome. We show here that bioluminescence of the dnaK-reporting Synechocystis transformant exhibited a daily oscillation which satisfied the criteria of circadian rhythms: persistence in constant conditions, phase resetting by lightdark signals, and temperature compensation of the period. The abundance of dnaK mRNA also exhibited a daily oscillation in constant conditions.

\section{MATERIALS AND METHODS}

Bacterial strains, media, cultures, enzymes, and manipulation of DNA. Wildtype cells of Synechocystis sp. strain PCC 6803 (gift of T. Ogawa) were maintained in BG-11 liquid medium (27) or on BG-11 agar that contained BG-11, 1 $\mathrm{mM}$ sodium thiosulfate, $10 \mathrm{mM} \mathrm{N}$-tris(hydroxymethyl)methyl-2-aminoethanesulfonic acid (TES, $\mathrm{pH} 8.2$ ), and 1.5\% Bacto-Agar (Difco Laboratories, Detroit, Mich.) at $30^{\circ} \mathrm{C}$ under continuous illumination (LL; intensity was about $35 \mu \mathrm{mol}$ $\mathrm{m}^{-2} \mathrm{~s}^{-1}$ from white fluorescent lamps). Bioluminescent reporter strains of the Synechocystis sp. constructed in this study were maintained in BG-11 medium supplemented with $40 \mu \mathrm{g}$ of spectinomycin sulfate per ml. Enzymes were purchased from Takara Shuzo Co. (Kyoto, Japan), New England Biolabs, Inc. (Beverly, Mass.), Promega Co. (Madison, Wis.), and Gibco BRL (Gaithersburg, Md.). Escherichia coli cells were maintained in Luria-Bertani broth (LB) or on LB agar that contained $1.2 \%$ agar in LB (Shouei Kanten Co., Tokyo, Japan). Plasmids were propagated in E. coli $\mathrm{HB} 101$ or DH5 $\alpha$. Handling of E. coli and manipulation of DNA for molecular cloning were carried out as described by Sambrook et al. (29).

Isolation of a genomic clone of the dnaK gene. We constructed a library of Synechocystis genomic DNA with the $\lambda$ DASH vector (Stratagene Cloning Systems, La Jolla, Calif.). A 2.1-kb DNA segment containing the coding region of the dnaK gene (6) was amplified by PCR with genomic DNA as a template and 


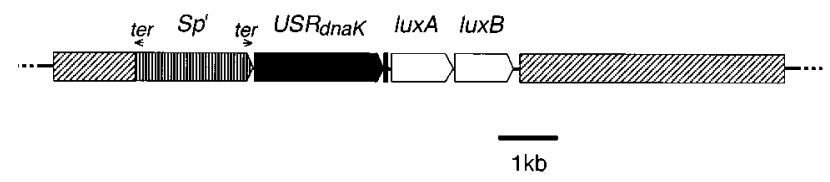

FIG. 1. Schematic representation of the $d n a K$ reporter plasmid pCF5. The open rectangles with arrowheads represent a promoterless segment of the luciferase lux $A B$ genes from $V$. harveyi and the $\Omega$ fragment carrying the spectinomycin/streptomycin resistance gene $\left(S p^{r}\right)$. An upstream region segment of the dnaK gene $\left(U S R_{d n a K}\right)$ is followed by an amino-terminal coding region of the dnaK gene (solid rectangle with arrowhead), which is fused out of frame with the lux $A$ gene. The arrows indicate the direction of transcription. The direction of the terminators (ter) of the $\Omega$ fragment is also shown by the arrows. The hatched boxes surrounding the $U S R_{\text {a }}: \because \operatorname{lux} A B$ gene fusion and the $\Omega$ fragment represent Synechocystis DNA sequences flanking the integration site in the chromosome. The pUC18 portions of the plasmid were not integrated into the chromosome and are not shown.

used as a hybridization probe. The $\lambda$ clone $\lambda$ DNAK2, which carries the dnaK gene, was isolated by plaque hybridization. A 7.6-kb Bam HI fragment of this $\lambda$ clone was subcloned into the BamHI site of pUC118 (37) to produce pDNAK. The $X c m I$ site of pDNAK was converted to a BamHI site by removing the protruding 3' nucleotide of the $X \mathrm{~cm} I$ terminus with T4 DNA polymerase (DNA blunting kit; Takara Shuzo), followed by the addition of a BamHI linker (d[pCG GATCCG]; Takara Shuzo) to produce pDNAKb.

Construction of reporter plasmids and Synechocystis reporter strains. pAM977 (gift of S. Golden) carries a $P_{p s b A I}:$ lux $A B$ gene fusion, namely, a promoter region of the Synechococcus psbAI gene $\left(P_{p s b A I}\right)$ connected to the coding region of the $V$. harveyi luciferase genes (luxAB) (14), and the $\Omega$ fragment, which contains the spectinomycin/streptomycin resistance gene (25). A 3.4-kb HindIII-EcoRI fragment of pAM977 which carried the $\Omega$ fragment and the $P_{p s b A I}:$ luxA gene portions of the $P_{p s b A I}:: l u x A B$ gene fusion was ligated with a 3.9-kb HindIII-EcoRI fragment of pLAV1 (gift of T. Baldwin) $(2,14)$ which carried the $\operatorname{lu} x B$ gene portions of the luxAB genes. A 4.6-kb HindIII-SmaI fragment of the resulting plasmid, pCF1, which carried the $\Omega$ fragment and the $P_{p s b A I}:: l u x A B$ gene fusion was converted to a $B g l \mathrm{II}$ fragment by the filling-in reaction with Klenow enzyme, followed by the addition of a $B g l \mathrm{II}$ linker (d[pC AGATCTG]) and digestion with $B g l$ II. The fragment was ligated into the unique $B g l$ II site of plasmid A71 (gift of T. Ogawa) (23); this site is located downstream of the Synechocystis $n d h B$ gene. The unique SplI site of the resulting plasmid (pCF2), located 54 bp upstream of the translation start site of the lux $A$ gene, was filled in with Klenow enzyme and converted to a BamHI site by the addition of a BamHI linker (d[pCGGATCCG]). A 230-bp BamHI fragment of this plasmid (pCF3) which contained the $p s b A I$ promoter segment was removed to produce a promoterless reporter plasmid (pCF4). Then, a 2.2-kb BamHI fragment of pDNAKb which carried a $2.16-\mathrm{kb}$ upstream region of the dnaK gene $\left(U_{S R_{\text {dnaK }}}\right)$ followed by a segment of the $d n a K$ amino-terminal coding region ( 13 codons plus 2 bp) was inserted into the unique BamHI site of pCF4. From this insertion, a plasmid which had the $d n a K$ upstream segment in the same direction as the lux $A B$ genes was selected as a dnaK reporter plasmid (pCF5). Another dnaK reporter plasmid which carried an 846-bp BstEII-XcmI segment of pDNAK was also constructed (pCF6). In this case, the BstEII- $X \mathrm{~cm}$ I fragment of pDNAK was converted with T4 DNA polymerase to a blunt-ended fragment which carried a shorter 805-bp upstream region of the dnaK gene followed by a segment of the dnaK amino-terminal coding region (13 codons plus $2 \mathrm{bp}$ ) and inserted into the unique Bam HI site of pCF4 which had been converted to a blunt-ended site with Klenow enzyme.

Wild-type Synechocystis cells were transformed with plasmids pCF4, pCF5, and pCF6 by the procedure described by Williams (38), and spectinomycin-resistant transformant colonies were selected. Transformants resulting from homologous recombination between the reporter plasmid and the host chromosome were selected by Southern blotting analysis. All transformants tested showed that the predicted recombination events had occurred. The CFC2 and CFC3 strains obtained by transformation with pCF5 and pCF6, respectively, carried the $U S R_{\text {dnaK }}:$ lux $A B$ (longer and shorter $U S R_{\text {dnaK }}$, respectively) and the $\Omega$ fragment at the $B g l$ II site of the chromosome and lacked vector sequences (Fig. 1). The CFC1 strain obtained by transformation with pCF4 carried the $\Omega$ fragment and the coding region of the luxAB gene but did not include the dnaK promoter segment. Three kinds of transformants showed normal morphology and growth rate. Thus, the insertion of the reporter constructs into the $B g l$ II site downstream of the $n d h B$ gene did not appear to be deleterious, so that this site can be used as a neutral integration site for exogenous DNA

Assay of the bioluminescence rhythm. Synechocystis cells were plated on BG-11 agar in LL (46 $\mu \mathrm{mol} \mathrm{m}{ }^{-2} \mathrm{~s}^{-1}$ from white fluorescent lamps) at $30^{\circ} \mathrm{C}$ for 2 days and then maintained for $12 \mathrm{~h}$ in the dark to synchronize the circadian clocks in the various cells of each colony. After the dark period, an agar block ( 15 by 15 by $7 \mathrm{~mm}$ ) with a patch of cells was cut out and transferred into a $20-\mathrm{ml}$ vial, and an open, sterile microcentrifuge tube containing $n$-decanal dissolved in vacuum pump oil at a concentration of $1.5 \%$ (vol/vol) was placed upright in the vial. Then, the vial was maintained in LL which was interrupted every $30 \mathrm{~min}$ for assay of the bioluminescence (25-min light and 5-min dark periods). We monitored the bioluminescence from cells on agar with a photomultiplier tube apparatus, as described previously $(13,14)$. Briefly, the vial was subjected to darkness for $100 \mathrm{~s}$ to allow chlorophyll fluorescence to decay, and the bioluminescence signal was accumulated for the next $100 \mathrm{~s}$ in the dark. After an additional exposure to darkness for $100 \mathrm{~s}$, the vial was returned to light conditions. Measurements were repeated every $30 \mathrm{~min}$ for 5 days. The light fluence rate was 46 $\mu \mathrm{mol} \mathrm{m} \mathrm{m}^{-2} \mathrm{~s}^{-1}$ during illuminated periods, and the temperature was maintained at $30^{\circ} \mathrm{C}$ unless otherwise specified. Brief, repetitive dark pulses during the assay protocol can have minor effects on circadian clocks but do not cause major interference (3).

The average level of bioluminescence of the $d n a K$ reporter strains CFC2 and CFC3 was about 1/20 of that expressed by the psbAI reporter Synechococcus strain (AMC149 [13]). Thus, it was necessary to enhance the sensitivity of our apparatus by using a low-noise photomultiplier tube (R2693P; Hamamatsu Photonics Co., Hamamatsu, Japan) at $900 \mathrm{~V}$, whose output was coupled to a photoncounting unit (C3866; Hamamatsu Photonics) to reduce thermal noise. The output pulses were counted by an electronic counter (model 7201; Iwatsu Co., Tokyo, Japan), and data were collected by data acquisition computers (PC-E200, Sharp Co., Osaka, Japan; Macintosh Plus, Apple Computer Co., Cupertino, Calif.). This modified apparatus was about 100 times more sensitive to light than the previously reported apparatus (14).

Mapping of the transcriptional initiation site of the $d n a K$ gene by the primer extension method. Wild-type Synechocystis cells were grown in liquid BG-11 medium and harvested at the logarithmic growth phase. RNA was extracted from cells as described by Mohamed and Jansson (22). Primer extension experiments were carried out by standard procedures (29), with SuperScript II RNase $\mathrm{H}^{-}$ reverse transcriptase (Gibco BRL) and nine primers: A (5'-CAGGAACGGTGATGACGGCTTGGGTAACGGTCTCC-3', +430 to +396; the first nucleotide $\mathrm{A}$ of the translational initiation codon of the $d$ naK gene is numbered +1 ), B (5'-GTACCGAGGTCAATCCCAACAACTTTTCCCATAAT-3', + 32 to -3), C (5'-AGTGACGGTAGGGGCTTAGTTCTCGGAAGTGCAGG-3', -107 to -141$)$, D (5'-GTCATCTTTGGTGGCGGAGCCCTTCTGCTGTT GAT-3', -287 to -321$)$, E (5'-ACTACCATCTGACCCACTTGCTGATT GAAGTTGGG-3', -573 to -607$)$, F (5'-GGAAAACATCTAGGGGGTCT TCTAGCAACCCACCT-3', -669 to -704$)$, G (5'-AGGGGATCGCCAGTAC GATTGATAATTAGGGCAGT-3', -705 to -739$)$, H (5'-GAATTGTCAGT GGTGAAGGAATCCGGCTCTAGTTG-3', -1131 to -1165$)$, and I (5'GGATTATTGAAATAGGCCTTGGGGGAAAGGGTCTC-3', $\quad-1188$ to $-1222)$. The $5^{\prime}$ ends of these primers were labeled with $\left[\gamma_{-}{ }^{32} \mathrm{P}\right] \mathrm{ATP}(>5,000$ $\mathrm{Ci} / \mathrm{mmol}$ ) by the $\mathrm{T} 4$ polynucleotide kinase reaction ( $\mathrm{T} 4$ polynucleotide kinase from Promega Co.). Ten micrograms of Synechocystis RNA and $4 \mathrm{ng}$ of ${ }^{32} \mathrm{P}$ labeled primer $\left(\sim 10^{5} \mathrm{cpm}\right)$ in $30 \mu$ of hybridization buffer were heated at $85^{\circ} \mathrm{C}$ for $10 \mathrm{~min}$ in a heat block for denaturation and then held overnight in the heat block without heating for annealing. RNA annealed with primer was recovered by ethanol precipitation and dried. The RNA and $5 \mu \mathrm{l}(1,000 \mathrm{U})$ of reverse transcriptase (Gibco BRL) in a 50- $\mu$ l reaction mixture for reverse transcriptase was incubated at $37^{\circ} \mathrm{C}$ for $2 \mathrm{~h}$ for the primer extension reaction. The reaction product was recovered by ethanol precipitation and applied to sequencing gels.

Northern (RNA) blotting analysis. Cells of the dnaK reporter Synechocystis sp. strain CFC2 were spread on BG-11 agar in 9-cm plastic dishes to yield about 5,000 colonies per dish. The dishes were incubated at $27^{\circ} \mathrm{C}$ in $\mathrm{LL}\left(38 \mu \mathrm{mol} \mathrm{m}{ }^{-2}\right.$ $\mathrm{s}^{-1}$ ). When the colonies had grown to $0.3 \mathrm{~mm}$ in diameter (growth for about 10 days), the dishes were subjected to darkness for $12 \mathrm{~h}$ to synchronize the circadian clocks among cells in the colonies and then returned to LL. The cells were harvested every $4 \mathrm{~h}$ in LL from four dishes by washing each dish with $2 \mathrm{ml}$ of BG-11 and collecting cell pellets by centrifugation, and then the pellets were immediately frozen in liquid nitrogen. RNA was extracted from each frozen sample as described by Mohamed and Jansson (22). RNA was subjected to electrophoresis on $1.0 \%$ formaldehyde-containing agarose gels $(0.5 \mu \mathrm{g}$ of total RNA was loaded per lane for dnaK mRNA gels and $4 \mu \mathrm{g}$ of total RNA was loaded per lane for luxAB mRNA gels), blotted onto positively charged nylon membranes (Boehringer Mannheim GmbH, Mannheim, Germany), and hybridized with a ${ }^{32} \mathrm{P}$-labeled probe specific for dnaK mRNA or luxAB mRNA by standard procedures (29). A 1.2-kb EcoRI fragment of the dnaK gene (the second nucleotide of codon 238 to the second nucleotide of codon 631) derived from pDNAK and a 1.2-kb PstI-Xcm I fragment of the luxAB genes (the second nucleotide of codon 185 of the lux $A$ gene to the first nucleotide of codon 233 of the $\operatorname{lux} B$ gene) derived from pLAV1 were labeled with $\left[\alpha-{ }^{32} \mathrm{P}\right] \mathrm{dCTP}(3,000$ $\mathrm{Ci} / \mathrm{mmol}$ ) by the nick translation reaction (random primer DNA labeling kit; Takara Shuzo) and used as hybridization probes. Hybridized bands were visualized and quantified with a Bio-Image Analyzer BAS2000 (Fuji Film Co., Kanagawa, Japan). The detection of radioactive bands with the BAS2000 is at least 20 times more sensitive than the usual X-ray film radioautographic detection method. The bioluminescence of the colonies on an agar block cut out from the dishes used for RNA extraction was also monitored for comparison by the procedure described above. 


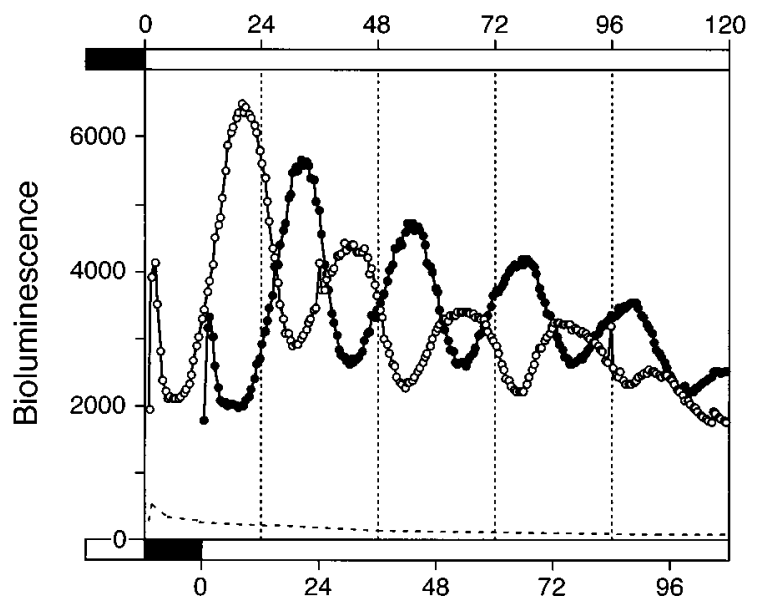

Hours in LL

FIG. 2. Bioluminescence rhythms of the dnaK reporter strain CFC2 in LL. Cells of the dnaK reporter strain $\mathrm{CFC} 2$ were cultured at $30^{\circ} \mathrm{C}$ in LL $(46 \mu \mathrm{mol}$ $\mathrm{m}^{-2} \mathrm{~s}^{-1}$ ) on BG-11 agar for 2 days and subjected to darkness for $12 \mathrm{~h}$ to synchronize the circadian clock. Then, they were returned to LL for monitoring of bioluminescence. Expression of bioluminescence from two CFC2 cultures that had been subjected to 12 -h dark periods which were $180^{\circ}$ out of phase are shown. The 12-h dark periods (solid bars) and the LL (open bars) are shown on the upper (for open circles) and lower (for solid circles) abscissae. The bioluminescence of CFC1 cells, which carried the promoterless lux $A B$ genes without the dnaK promoter segment, is also shown as a control (dashed line). The light regimen for the $\mathrm{CFC} 1$ culture is as shown on the upper abscissa. The vertical axis indicates bioluminescence intensity as photon counts per second. The first transient peaks of the bioluminescence should be ignored because they were artifacts of the saturation and penetration into cells of $n$-decanal vapor, which was added at the ends of the dark periods.

\section{RESULTS AND DISCUSSION}

Bioluminescence rhythms of the dnaK reporter Synechocystis strains. To monitor the expression of the $d n a K$ gene (6) in Synechocystis sp. strain PCC 6803 as bioluminescence, we used the same procedure used for monitoring the expression of the psbAI gene in another unicellular cyanobacterium, Synechococcus sp. strain PCC $7942(13,14)$ except that our current apparatus is more sensitive to light (see Materials and Methods).

We first constructed a dnaK reporter plasmid, pCF4, which carries a $U S R_{\text {dna }}:: l u x A B$ gene fusion, namely, a $2.16-\mathrm{kb}$ upstream region segment of the $d n a K$ gene fused to a promoterless segment of the luciferase lux $A B$ gene set from $V$. harveyi (Fig. 1). In this construct, the expression of the lux $A B$ genes is under the control of the upstream region of the $d n a K$ gene including the $d n a K$ promoter, since activity from the upstream flanking region of the $U S R_{d n a K}$ in the dnaK reporter plasmid is blocked by a transcriptional terminator located in the $\Omega$ fragment. To construct a dnaK reporter Synechocystis strain (CFC2), we transformed wild-type Synechocystis cells with the reporter plasmid and integrated the reporter gene without vector sequences into a specific integration site of the chromosome, a $B g l$ II site downstream of the $n d h B$ gene (23), via homologous recombination. Growth of the dnaK reporter strain was normal and thus was apparently unaffected by the insertion of the reporter gene into this integration site in the chromosome.

Figure 2 shows the time course of bioluminescence measured from the dnaK reporter strain maintained in LL at $30^{\circ} \mathrm{C}$. To synchronize a presumed circadian clock, we exposed two cultures to dark intervals ( $12 \mathrm{~h}$ in duration) that were $180^{\circ}$ out of phase before the onset of LL and then returned them to LL to monitor their bioluminescence. The bioluminescence from both cultures oscillated with a period of $22.4 \pm 0.4 \mathrm{~h}$ (data from four separate experiments) but with opposite phases, which depended upon the timing of the prior 12-h dark interval. The peak of bioluminescence occurred at about $19.1 \mathrm{~h}$ after the onset of LL. The peak-to-trough ratio of the rhythms was about 3 on the first day of LL, but it decreased gradually. These results indicate that the bioluminescence rhythm was the expression of an endogenous circadian clock, because the rhythms continued under constant conditions with a period of about 1 day, and also indicate that the phase of the rhythm was entrained by the 12-h dark intervals which preceded LL.

As a control, we also monitored the bioluminescence from a control reporter strain (CFC1) which had only a promoterless segment of the lux $A B$ genes and lacked an exogenous promoter segment (Fig. 2). The level of the bioluminescence was about $1 / 40$ of the average level of the bioluminescence from the dnaK reporter strain $\mathrm{CFC} 2$, and as predicted, the bioluminescence showed no obvious oscillation. This result indicates that the bioluminescence rhythm of the dnaK reporter strain was mainly controlled by the upstream region of the dnaK gene.

The 2.16-kb upstream region of the dnaK gene used here as the $U S R_{\text {dnaK }}$ also carried an uncharacterized upstream open reading frame, ORF70-5 (6), which is adjacent to the $5^{\prime}$ region of the dnaK gene and may also carry additional upstream genes. Thus, CFC2 reported the dnaK gene expression from an additional upstream promoter(s) as well as that controlled by the major dnaK promoter. To monitor the activity of the major $d n a K$ promoter, we constructed another $d n a K$ reporter strain, $\mathrm{CFC} 3$, which was the same as $d n a K$ reporter strain $\mathrm{CFC} 2$ except that strain CFC3 carried an 846-bp upstream region segment of the $d n a K$ gene as a promoter. This segment carries the $3^{\prime}$ portion of the ORF70-5 but lacks the $5^{\prime}$ portion of the $O R F 70-5$, including its promoter region. We monitored the bioluminescence from strain CFC3 (Fig. 3b). The level, amplitude, and waveform of the bioluminescence rhythm from strain CFC3 were very similar to those of the bioluminescence rhythm from strain CFC2 (Fig. 3a and b). This result indicates that the bioluminescence rhythm of the $d n a K$ reporter strains

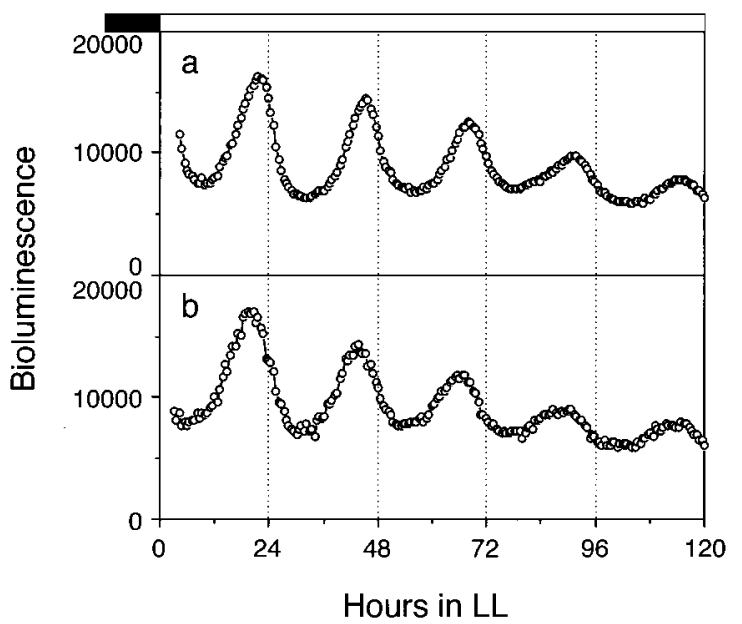

FIG. 3. Bioluminescence rhythms of the dnaK reporter strains CFC2 and CFC3 in LL. Cells of the dnaK reporter strains CFC2 (a) and CFC3 (b) were subjected to darkness for $12 \mathrm{~h}$ to synchronize the circadian clock. Then, they were returned to LL for monitoring of bioluminescence. The light regimen is shown on the upper abscissa. Other conditions were the same as described in the legend to Fig. 2 except that the light intensity was $23 \mu \mathrm{mol} \mathrm{m} \mathrm{m}^{-2} \mathrm{~s}^{-1}$. 
was mainly controlled by a major $d n a K$ promoter which should be located in the 846-bp upstream region of the dnaK gene.

Mapping of the transcriptional initiation site of the dnaK gene by primer extension. Northern blotting experiments demonstrated two dnaK transcripts, a 2.2-kb major transcript and a minor longer transcript (see Fig. 5d; the minor band is not visible in Fig. 5d), as described previously (6). We mapped the transcriptional initiation site of the dnaK gene by the primer extension method with nine primers (A to I). We found a single primer extension band which corresponds to an initiation site at $\mathrm{T}$ which is located at base 1278 upstream from the translational initiation codon of the dnaK gene by primer $\mathrm{H}$ $(-1131$ to -1165$)$ (the nucleotide number of the $\mathrm{T}$ is -1278 when the first nucleotide $A$ of the translational initiation codon of the dnaK gene is numbered +1$)$. This transcriptional initiation site probably corresponds to a minor longer dnaK transcript. We also mapped a major $d n a K$ transcriptional initiation site which should be located on the 846-bp upstream region of the dnaK gene by using primers $\mathrm{A}(+430$ to +396$), \mathrm{B}(+32$ to $-3), C(-107$ to -141$), D(-287$ to -321$), E(-573$ to -607$)$, F ( -669 to -704$)$, and $G(-705$ to -739$)$, but we could not find any clear primer extension band. When we used primer $\mathrm{E}$, we detected a doublet band in primer extension and stop bands in all lanes in a sequencing gel which corresponded to the double band in primer extension. Thus, the primer extension reaction might be hindered by a possible secondary structure of a major shorter $d n a K$ transcript or for unknown reasons.

Temperature compensation of the bioluminescence rhythm. Another key characteristic of circadian rhythms is the temperature compensation of the period. We examined the effects of temperature on the period length of the bioluminescence rhythm. After synchronization of the circadian clock by exposure to a 12-h dark period, we maintained cells of the dnaK reporter strain $\mathrm{CFC} 2$ at 25,30 , or $35^{\circ} \mathrm{C}$ in $\mathrm{LL}$ and monitored their bioluminescence. Figure $4 \mathrm{a}$ shows the time course of the bioluminescence at the three different temperatures in LL. The period lengths of the rhythm were almost constant and were found to be $22.6 \pm 0.1,22.4 \pm 0.4$, and $21.2 \pm 0.4 \mathrm{~h}$ at 25,30 , and $35^{\circ} \mathrm{C}$, respectively. The rhythms were more obvious at lower temperatures, especially for the first 3 days. Even at $35^{\circ} \mathrm{C}$, however, clear peaks and troughs of the rhythm were observed, although the amplitude of the rhythm was low. We plotted the period length against temperature (Fig. 4b) and calculated the $Q_{10}$ value for frequency (1/period) to be 1.1 (the period decreased by $1.4 \mathrm{~h}$ when the temperature increased from 25 to $35^{\circ} \mathrm{C}$ ). This indicates that the period length of the bioluminescence rhythm was well compensated for against changes in ambient temperature.

Therefore, the bioluminescence rhythm of the dnaK reporter strain CFC2 satisfied the three criteria for circadian rhythms: persistence in constant conditions (Fig. 2), phase resetting by light-dark signals (Fig. 2), and temperature compensation of the period (Fig. 4). Thus, we can conclude that both cyanobacterial species Synechocystis sp. strain PCC 6803 and Synechococcus sp. strain PCC 7942 (14) have circadian pacemakers that exhibit the same basic properties as those of eucaryotic organisms.

Rhythmic changes in the abundance of dnaK mRNA in LL. The data described above suggest that the expression of the dnaK gene was controlled by the circadian clock. To test this possibility more directly, we examined the changes in the abundance of dnaK mRNA and that of luxAB mRNA of the dnaK reporter strain CFC2 during LL. After synchronization of the circadian clock by a 12-h dark interval, we maintained cells of the dnaK reporter strain at $27^{\circ} \mathrm{C}$ in LL and extracted RNA from cells harvested at 4-h intervals. We estimated the relative
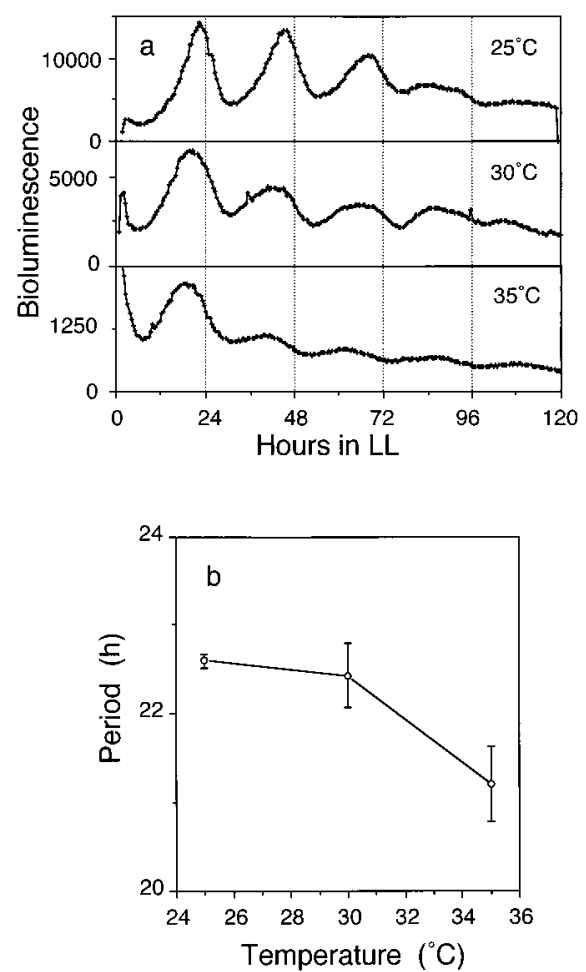

FIG. 4. Temperature compensation of the period of the bioluminescence rhythms. After the 12 -h dark period to synchronize the circadian clock, CFC2 cells were maintained in LL $\left(46 \mu \mathrm{mol} \mathrm{m} \mathrm{m}^{-2} \mathrm{~s}^{-1}\right)$ at three different temperatures $\left(25,30\right.$, and $\left.35^{\circ} \mathrm{C}\right)$ to have their bioluminescence monitored. (a) Rhythms of bioluminescence at the three different temperatures. (b) Periods of the rhythms plotted as a function of temperature. The bars indicate the standard deviation of at least three replicate experiments. Other conditions were the same as described in the legend to Fig. 2.

abundance of $d n a K$ mRNA and of $\operatorname{lu} x A B$ mRNA at various times in LL by Northern blotting analysis with ${ }^{32} \mathrm{P}$-labeled probes.

The dnaK mRNA bands were two bands, a longer discrete band with a smear, which corresponds to dnaK mRNA and its degradation products, and a shorter uncharacterized band (Fig. 5d). The shorter band was a discrete band and might be a processed dnaK mRNA or an antisense transcript of the $d n a K$ gene. The $\operatorname{lux} A B$ mRNA bands were smears (Fig. 5e).

Figures $5 \mathrm{a}$ and $\mathrm{b}$ show the rhythmic changes in the abundance of $d n a K$ mRNA and $l u x A B$ mRNA during LL. We also included a profile of the bioluminescence rhythm for comparison (Fig. 5c). We found that both mRNAs showed daily rhythmic changes in their abundance. The peak-to-trough amplitude (the mean ratio of the first peak value to the second trough value obtained from two or three separate experiments) of the rhythms in dnaK mRNA and $l u x A B$ mRNA was about 1.8 and 3.3 , respectively. The peaks of the rhythm in the lux $A B$ mRNA abundance occurred at approximately the same times as those of the bioluminescence rhythm (Fig. 5c), that is, at 20 and $44 \mathrm{~h}$. However, the peaks of the rhythm in the dnaK mRNA abundance were delayed by 4 to $8 \mathrm{~h}$ compared with those of the rhythm in luxAB mRNA abundance. The abundance differed greatly between $d n a K$ mRNA and lux $A B$ mRNA. The average abundance of $\operatorname{lu} x A B$ mRNA was about 10 -fold less than that of dnaK mRNA. This difference in phase of the mRNA rhythms and the low abundance of $\operatorname{lu} x A B$ mRNA may reflect differences in the decay rates of the two mRNAs. 


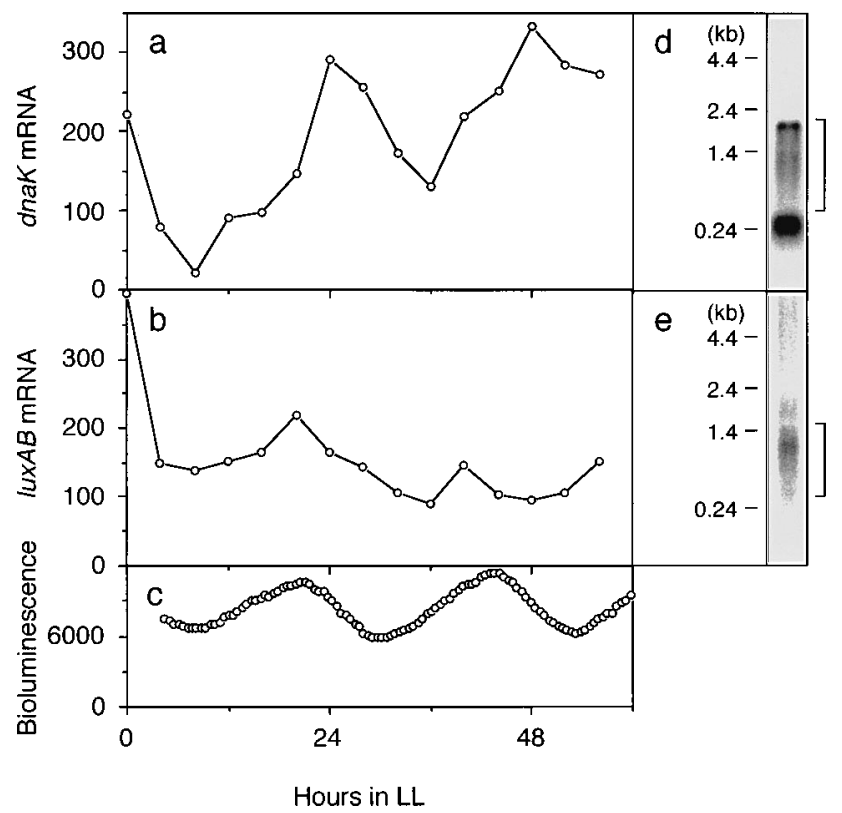

FIG. 5. Rhythmic changes in the abundance of $d n a K$ mRNA in LL. CFC2 cells were grown on BG-11 agar in plastic dishes at $27^{\circ} \mathrm{C}$ in $\mathrm{LL}\left(38 \mu \mathrm{mol} \mathrm{m}{ }^{-2}\right.$ $\mathrm{s}^{-1}$ ) until the colonies grew to $0.3 \mathrm{~mm}$ in diameter. Then, the cells were subjected to darkness for $12 \mathrm{~h}$ to synchronize the circadian clock and thereafter maintained in LL. The cells from aliquots of dishes were harvested every $4 \mathrm{~h}$ in LL, and RNA was extracted from each sample. The abundance of $d n a K$ mRNA and lux $A B$ mRNA was estimated by Northern blotting analyses with ${ }^{32} \mathrm{P}$-labeled probes specific for dnaK mRNA and luxAB mRNA, respectively. Hybridized bands were visualized by a Bio-Image Analyzer BAS2000 (d, dnaK mRNA; e, lux $A B$ mRNA), and the relative radioactivity of the bands was also quantified by BAS2000 and plotted against time in LL. Estimated regions of the bands with smear are also shown by the lines in panels $\mathrm{d}$ and e. Bioluminescence from the colonies on a BG-11 agar block is also shown for comparison. (a) dnaK mRNA; (b) luxAB mRNA; (c) bioluminescence.

These data indicate that a circadian clock controls the activity of the $d n a K$ promoter, resulting in daily rhythmic changes in both dnaK mRNA and luxAB mRNA levels. Although we have not yet determined the intracellular level of bacterial luciferase, the bioluminescence rhythm exhibited by the dnaK reporter strain probably results from rhythmic changes in the intracellular level of luciferase. The $p s b A I$ reporter derivative of Synechococcus sp. strain PCC 7942 (AMC149) showed daily changes in the intracellular level of luciferase $(14,19)$. As discussed previously (14), the bioluminescence reaction catalyzed by bacterial luciferase can be influenced not only by changes in the intracellular level of the luciferase but also by changes in the levels of its substrates, a reduced form of flavin mononucleotide $\left(\mathrm{FMNH}_{2}\right), n$-decanal, and $\mathrm{O}_{2}$. The effective level of $n$-decanal is expected to be constant because it was supplied exogenously, but the levels of $\mathrm{FMNH}_{2}$ and $\mathrm{O}_{2}$ are unknown. Therefore, other steps for generating bioluminescence could also be possibly controlled by the circadian clock, e.g., degradation of $\operatorname{lu} x A B$ mRNA, translation of $l u x A B$ mRNA (de novo synthesis of luciferase), degradation of luciferase, and metabolic control of the intracellular levels of $\mathrm{FMNH}_{2}$ and/or $\mathrm{O}_{2}$. However, because the phase of bioluminescence rhythms reported for various genes in Synechocystis sp. strain PCC 6803 (as well as in Synechococcus sp. strain PCC 7942 [20]) differs from gene to gene (unpublished data; see also below), it is not easy to determine at which step(s) the circadian clock is regulating bioluminescence.

We demonstrate here that the circadian clock controls the expression of the dnaK gene in this Synechocystis strain. At present, we do not know how the clock controls the activity of the $d n a K$ promoter. Our monitoring system with the luciferase $\operatorname{lu} x A B$ genes as a reporter should be useful in solving this problem at the molecular level. Because DnaK has a key role in controlling the expression of other heat shock (or stress) genes in E. coli $(34,36)$, such downstream genes are also likely to be controlled by the clock via DnaK. We want to verify this circadian control cascade by the same method as described here. Circadian control of the expression of the $d n a K$ gene may be involved in the adaptation to 24-h high-temperature/lowtemperature cycles resulting from the day-night cycles on the earth. Consistent with that idea, the circadian clock in the Synechocystis strain can be reset not only by light-dark cycles but also by low-temperature/high-temperature cycles (unpublished data). An interesting question is whether heat shock proteins, including DnaK, are involved in the temperaturesensing mechanism of the circadian clock.

There are several differences between the bioluminescence rhythms of the reporter strains in Synechocystis sp. strain CFC2 versus Synechococcus sp. strain AMC149 $(13,14)$. The period of CFC2 $(22.4 \mathrm{~h})$ is shorter than that of AMC149 $(24 \mathrm{~h})$ by 1.6 $\mathrm{h}$ at $30^{\circ} \mathrm{C}$. We know that this difference in period is not due to the difference between using promoters from the dnaK and $p s b A I$ genes. For example, we have examined many other genes in Synechococcus sp. strain PCC 7942 (20) and several other genes in Synechocystis sp. strain PCC 6803 (unpublished data) and have found that although the peak positions of bioluminescence reported for the promoters of different genes are different, the period of the bioluminescence rhythms is specific to each species: $22.4 \mathrm{~h}$ in the Synechocystis strain and $24 \mathrm{~h}$ in the Synechococcus strain. The peak of the rhythm of CFC2 occurred $19.1 \mathrm{~h}$ after the onset of LL, which corresponds to $3 \mathrm{~h}$ before subjective dawn ("dawn" in continuous conditions) when cells have been entrained under 24-h light-dark cycles, while the peak of the rhythm expressed by AMC149 occurs $12 \mathrm{~h}$ after the onset of LL, which corresponds to subjective dusk ("dusk" in continuous conditions). The rhythm of CFC2 persisted for only 5 days, whereas that of AMC149 continued for more than 2 weeks. Thus, the optimal conditions for persistence of the rhythms may be different between these two species, or desynchronization of the clocks of individual cells in CFC2 might occur earlier than in AMC149. Furthermore, the average level of bioluminescent signals obtained from CFC2 was only $1 / 20$ of that from AMC149. At present, it is not feasible to monitor bioluminescence rhythms of individual colonies of CFC2 on agar plates by an automated monitoring system which has recently been developed for screening for clock mutants of AMC149 (15).

It has become evident that a circadian clock exists in various kinds of cyanobacteria, whether they are unicellular or filamentous, nitrogen fixing or nonfixing. The two species characterized by us, Synechocystis sp. strain PCC 6803 and Synechococcus sp. strain PCC 7942, are unicellular cyanobacteria that do not fix nitrogen. Cell division of the unicellular cyanobacterium Synechococcus sp. strain WH7803, which does not fix nitrogen, is controlled by a circadian clock (35). In the nitrogen-fixing unicellular cyanobacteria Synechococcus sp. strain Miami BG 043511 (21), Synechococcus sp. strain RF-1 $(5,10)$, and Cyanothece sp. strain ATCC 51142 (31), metabolic activities such as nitrogen fixation, amino acid uptake, photosynthetic oxygen evolution, and carbohydrate granule formation or cell division are also under the control of the clock. A circadian clock also exists in nitrogen-fixing filamentous cyanobacteria, such as Oscillatoria sp. strain 23 (33), Trichodesmium thiebautii (28), and Anabaena sp. strain PCC 7120, as we recently discovered by using the luciferase reporter system 
described here (10a). In relation to the evolution of the circadian clock, it will be interesting to determine whether organisms that have a lower or higher organization than that of cyanobacteria but are not too distantly related to cyanobacteria have a similar circadian clock. We should be able to address this question by using our luciferase reporter system.

\section{ACKNOWLEDGMENTS}

We thank T. Ogawa (Institute of Physical and Chemical Research, Saitama, Japan), S. Golden (Texas A\&M University), and T. Baldwin (Texas A\&M University) for providing us with plasmid A71 and the Synechocystis strain, plasmid pAM977, and plasmid pLAV1, respectively. We also thank M. Sugita (Center for Gene Research, Nagoya University, Nagoya, Japan) for valuable advice on primer extension experiments. We are grateful for helpful discussion with C. H. Johnson (Vanderbilt University), S. Golden, and S. Itoh (National Institute for Basic Biology, Japan). We also thank C. H. Johnson and S. Golden for critical reading of the manuscript.

This research was supported by grants from the Japanese Ministry of Education, Science and Culture (06807029 and 06670093), the Nissan Foundation (Tokyo, Japan), and the Yamada Foundation (Osaka, Japan).

\section{REFERENCES}

1. Anderson, S. L., and L. McIntosh. 1991. Light-activated heterotrophic growth of the cyanobacterium Synechocystis sp. strain PCC 6803: a bluelight-requiring process. J. Bacteriol. 173:2761-2767.

2. Baldwin, T. O., T. Berends, T. A. Bunch, T. F. Holzman, S. K. Rausch, L. Shamansky, M. L. Treat, and M. M. Ziegler. 1984. Cloning of the luciferase structural genes from Vibrio harveyi and expression of the bioluminescence in Escherichia coli. Biochemistry 23:3663-3667.

3. Broda, H., V. D. Gooch, W. Taylor, N. Aiuto, and J. W. Hastings. 1986. Acquisition of circadian bioluminescence data in Gonyaulax and an effect of the measurement procedure on the period of the rhythm. J. Biol. Rhythms 1:251-263.

4. Bünning, E. 1973. The physiological clock. Springer-Verlag, New York.

5. Chen, T.-H., T.-L. Chen, L.-M. Hung, and T.-C. Huang. 1991. Circadian rhythm in amino acid uptake by Synechococcus RF-1. Plant Physiol. 97:5559.

6. Chitnis, P. R., and N. Nelson. 1991. Molecular cloning of the genes encoding two chaperon proteins of the cyanobacterium Synechocystis sp. PCC 6803. J. Biol. Chem. 266:58-65.

7. Cornelius, G., and L. Rensing. 1986. Circadian rhythm of heat shock protein synthesis of Neurospora crassa. Eur. J. Cell Biol. 40:130-132.

8. Craig, E. A., J. S. Weissman, and A. L. Horwich. 1994. Heat shock proteins and molecular chaperones: mediators of protein conformation and turnover in the cell. Cell 78:365-372.

9. Gamer, J., H. Bujard, and B. Bukau. 1992. Physical interaction between heat shock proteins DnaK, DnaJ, and GrpE and the bacterial heat shock transcription factor $\sigma^{32}$. Cell 69:833-842.

10. Grobbelaar, N., T. C. Huang, H. Y. Lin, and T. J. Chow. 1986. Dinitrogenfixing endogenous rhythm in Synechococcus RF-1. FEMS Microbiol. Lett. 37:173-177.

10a.Ishiura, M., and T. Kondo. Unpublished data.

11. Johnston, R. N., and B. L. Kucey. 1988. Competitive inhibition of $h s p 70$ gene expression causes thermosensitivity. Science 242:1551-1554.

12. Kappen, L., and R. Lösch. 1984. Diurnal patterns of heat tolerance in relation to CAM. Z. Pflanzenphysiol. 114:87-96.

13. Kondo, T., and M. Ishiura. 1994. Circadian rhythms of cyanobacteria: monitoring the biological clocks of individual colonies by bioluminescence. J. Bacteriol. 176:1881-1885.

14. Kondo, T., C. A. Strayer, R. D. Kulkarni, W. Taylor, M. Ishiura, S. S. Golden, and C. H. Johnson. 1993. Circadian rhythms in prokaryotes: luciferase as a reporter of circadian gene expression in cyanobacteria. Proc. Natl. Acad. Sci. USA 90:5672-5676.
15. Kondo, T., N. F. Tsinoremas, S. S. Golden, C. H. Johnson, S. Kutsuna, and M. Ishiura. 1994. Circadian clock mutants of cyanobacteria. Science 266: 1233-1236.

16. Kusukawa, N., and T. Yura. 1988. Heat shock protein GroE of Escherichia coli: key protective roles against thermal stress. Genes Dev. 2:874-882.

17. Landry, J., P. Chrétien, H. Lambert, E. Hickey, and L. A. Weber. 1989. Heat shock resistance conferred by expression of the human HSP27 gene in rodent cells. J. Cell Biol. 109:7-15.

18. Laude, H. H. 1939. Diurnal cycle of heat resistance in plants. Science 89: $556-557$.

19. Liu, Y., S. S. Golden, T. Kondo, M. Ishiura, and C. H. Johnson. 1995. Bacterial luciferase as a reporter of circadian gene expression in cyanobacteria. J. Bacteriol. 177:2080-2086.

20. Liu, Y., N. F. Tsinoremas, C. H. Johnson, N. V. Lebedeva, S. S. Golden, M. Ishiura, and T. Kondo. 1995. Circadian orchestration of gene expression in cyanobacteria. Genes Dev. 9:1469-1478.

21. Mitsui, A., S. Kumazawa, A. Takahashi, H. Ikemoto, S. Cao, and T. Arai. 1986. Strategy by which nitrogen-fixing unicellular cyanobacteria grow photoautotrophically. Nature (London) 323:720-722.

22. Mohamed, A., and C. Jansson. 1989. Influence of light on accumulation of photosynthesis-specific transcripts in the cyanobacterium Synechocystis 6803. Plant Mol. Biol. 13:693-700.

23. Ogawa, T. 1991. A gene homologous to the subunit-2 gene of NADH dehydrogenase is essential to inorganic carbon transport of Synechocystis PCC 6803. Proc. Natl. Acad. Sci. USA 88:4275-4279.

24. Otto, B., B. Grimm, P. Ottersbach, and K. Kloppstech. 1988. Circadian control of the accumulation of mRNAs for light- and heat-inducible chloroplast proteins in pea (Pisum sativum L.). Plant Physiol. 88:21-25.

25. Prentki, P., and H. M. Krish. 1984. In vitro insertional mutagenesis with a selectable DNA fragment. Gene 29:303-313.

26. Rikin, A. 1992. Circadian rhythm of heat resistance in cotton seedlings: synthesis of heat-shock proteins. Eur. J. Cell Biol. 59:160-165.

27. Rippka, R., J. Deruelles, J. B. Waterbury, M. Herdman, and R. Y. Stanier. 1979. Generic assignments, strain histories and properties of pure cultures of cyanobacteria. J. Gen. Microbiol. 111:1-61.

28. Roenneberg, T., and E. J. Carpenter. 1993. Daily rhythm of $\mathrm{O}_{2}$-evolution in the cyanobacterium Trichodesmium thiebautii under natural and constant conditions. Mar. Biol. 117:693-697.

29. Sambrook, J., E. F. Fritsch, and T. Maniatis. 1989. Molecular cloning: a laboratory manual, 2nd ed. Cold Spring Harbor Laboratory Press, Cold Spring Harbor, N.Y.

30. Sanchez, Y., and S. L. Lindquist. 1990. HSP104 required for induced thermotolerance. Science 248:1112-1115.

31. Schneegurt, M. A., D. M. Sherman, S. Nayar, and L. A. Sherman. 1994 Oscillating behavior of carbohydrate granule formation and dinitrogen fixation in the cyanobacterium Cyanothece sp. strain ATCC 51142. J. Bacteriol. 176:1586-1597.

32. Schwemmle, B., and O. L. Lange. 1959. Endogen-tagesperiodische Schwankungen der Hitzeresistenz bei Kalanchoë blossfeldiana. Planta 53: 134-144.

33. Stal, L. J., and W. E. Krumbein. 1985. Nitrogenase activity in the nonheterocystous cyanobacterium Oscillatoria sp. grown under alternating lightdark cycles. Arch. Microbiol. 143:67-71.

34. Straus, D., W. Walter, and C. A. Gross. 1990. DnaK, DnaJ, and GrpE heat shock proteins negatively regulate heat shock gene expression by controlling the synthesis and stability of $\sigma^{32}$. Genes Dev. 4:2202-2209.

35. Sweeney, B. M., and M. B. Borgese. 1989. A circadian rhythm in cell division in a prokaryote, the cyanobacterium Synechococcus WH7803. J. Phycol. 25:183-186.

36. Tilly, K., N. McKittrick, M. Zylicz, and C. Georgopoulos. 1983. The dnaK protein modulates the heat-shock response of Escherichia coli. Cell 34:641646.

37. Vieira, J., and J. Messing. 1987. Production of single-stranded plasmid DNA. Methods Enzymol. 153:3-11.

38. Williams, J. G. K. 1988. Construction of specific mutations in photosystem II photosynthetic reaction center by genetic engineering methods in Synechocystis 6803. Methods Enzymol. 167:766-778.

39. Yamamori, T., and T. Yura. 1982. Genetic control of heat-shock protein synthesis and its bearing on growth and thermal resistance in Escherichia coli K-12. Proc. Natl. Acad. Sci. USA 79:860-864. 